\title{
Analysis on Linguistic Features and Impact of Web-Chat English
}

\author{
ZHU Ya-dong, ZHU Kui \\ Sichuan Agricultural University, Ya’an, China
}

\begin{abstract}
Chatting online, a new way of communication, is becoming more and more popular. Web-chat English, a new variety of English, comes into our life as the result of the fast development of the Internet. As a special kind of English, web-chat English reflects new changes of our modern life with its distinctive features. This study mainly analyses features of the new variety from linguistic aspects: form level, lexical level, and syntactic level. It also discusses the impact that the web-chat English has brought us in our daily communication. Through the analysis and discussion, the study is aimed at giving readers an overall understanding of web-chat English.
\end{abstract}

Keywords: web-chat English, linguistic features, impact

\section{Introduction}

Language and information are two important parts in modern society. Where there is communication, there will be information and language. The 21 st century is an information age. The development of the Internet is a great milestone in the history of mankind, which is also the embryonic form of the future information superhighway. From then on, human beings enter an unprecedented information society. With the rapid development of computer technology, the Internet, as a communication medium, has become an important part in modern people's life with its high efficiency, high spreading speed, and the large capacity of information storage. Now the Internet has brought about changes into many aspects of people's life. More and more people attach great importance to the power of the Internet. Surfing the Internet has become more and more popular, especially chatting online, which is much convenient and efficient.

Under this circumstance, web-chat English, a new variety of language has come into people's life. It is a special kind of English, used in the chatting room or through chatting tools on the Internet. It gets close to our social life and reflects new changes of modern society. Just as the linguist David Crystal states in his book Language and the Internet, "If the Internet is a revolution, therefore, it is likely to be a new linguistic revolution" (Cristal, 2001). From his words, we can see that it is important and necessary to have a research on the popular web-chat English. Based on the previous study, this study pays more attention to the formation and features of web-chat English and attempts to enable readers to have a better understanding of this new variety of English.

\section{The Formation of Web-Chat English}

Language is the most important tool for human beings' communication. It is a social phenomenon which

ZHU Ya-dong, lecturer, School of Literature and Law, Sichuan Agricultural University.

ZHU Kui, associate professor, School of Literature and Law, Sichuan Agricultural University. 
exists for serving the society (QI, 1985). Meanwhile, different social systems determine the various languages in the world. Even for primitive tribes, they have their own languages. All of human's social life is totally or at least partly reflected in the language. Therefore, the development of society is bound to cause changes in the language. Since our society develops all the time, the development of language never stops. The social development enriches and perfects our language. In turn, language as a necessary part of social life greatly promotes the development of society. Without language, it is difficult to imagine the social communication go on. In fact, language can be regarded as the storage of human's labor practice. It helps people make manufacture activities, transform the nature, develop the culture, and spread knowledge. It is an essential tool to maintain the contemporary social life and one of the main powers to promote the social development.

The development of the Internet is a great milestone in the history of mankind, which is also the embryonic form of the future information superhighway. It is the product of rapid development of computer technology and can be traced back to 1962. At that time, computer scientists and engineers working for the American military attempted to connect computers with one another from different location. In 1969, an experimental system known as "Arpanet" was used to connect computers in some American universities. In 1972, emails were first used on the Internet. The rapid development of the Internet first originated from the establishment of United States NSF (National Science Foundation). On January 1st, 1983, Arpanet and other major networks were switched to TCP/IP (Transmission Control Protocol/Internet Protocol) program. With this connection, information exchange among various networks became possible. Just for the breakthrough, that day was regarded as the birthday of the Internet. After that, the Internet gradually came into people's daily life. With the rapid development of the Internet, more and more people are used to using it to deal with public and personal affairs, such as shopping online, chatting, playing games, and sending emails. A virtual place on the Internet, the chatting room becomes a good place for people to communicate and relax in the modern busy life. Then, web-chat English, a new variety of language comes into being and is gradually accepted by more and more people.

\section{Analysis on Features of Web-Chat English}

\section{The Definition of Web-Chat English}

When it comes into the 21 st century, the popularity of the Internet has guided us into information age. Due to the growth of netizens as well as the advantages of chatting online, web-chat English becomes quite popular. More and more scholars begin to pay attention to this new form of English. Since web-chat English is a new subject not widely accepted in the circle of linguistics, it is not easy to define it accurately. Some people consider it as a set of conventions for the language applied to communication on the Internet. Some hold the view of classifying it as a collection of words, idioms, slangs, terms, abbreviations, and similes that are characteristic of online communication.

In simple words, web-chat English is the special English used in the chatting room for communication. As we know, language exists for people's demands of communication. Web-chat English, as a new member of English family, also follows the regularity and gives services to the online chatters (Cherny, 1999). In details, it can be understood as such a new variety widely used on the internet in modern society, with its own features on form, vocabulary, syntax, and content unique to the Internet. 


\section{Features of Web-Chat English at Form Level}

To learn a new language, we should first learn about its form. Web-chat English has its features on form different from traditional English as follows. First, to seek the brevity and convenience, participants always ignore the capitalization in a sentence, which is a common phenomenon in the chatting room. This is probably due to speakers' purpose of saving energy and time. Most of chatting participants want to send his/her messages as quickly as possible and they regard it is a waste of time to press the "Capslock" key to type a capital letter. Besides, the omission of capitalization cannot change the meaning of sentences at all. So capital letters are not considered so important in web-chat English. The omission of capitalization is the first obvious characteristics of web-chat English at form level. In spite of the frequent omission of capitalization, capitalized and bold forms of words are often used to emphasize or express one's powerful feelings to draw other's attention. For example, one may use the sentence "TOO MUCH USE OF CAPITALS IS BAD MANNERS" for expressing his/her strong dissatisfaction by using the capitals. The bold words or phrases in the chatting room stand for the key point, to which you should pay more attention.

Secondly, the deviant use of punctuation is one of the most remarkable features in the chatting room. In fact, the original function of punctuation is to help readers make clear understanding of the structure and meaning of a sentence. Generally speaking, the repeated punctuation marks are seldom used in our traditional language. However, this phenomenon is very familiar to every participant in the chatting room. Sometimes, in order to express strong emotions or feelings, we may use the repeated marks which can give each other a fresh feeling and make the communication more interesting.

Here are some examples (see Examples 1-3):

Example (1) Oh yeah!!!! (Feeling of happiness or satisfaction)

Example (2) No way!!!!! (Emphasis that you can't)

Example (3) What?????? (Feeling of surprise)

Meanwhile, punctuation marks are omitted in most cases in online chatting. To seek for efficiency, the sentences of web-chat English are always short and easy for understanding. Therefore, the function of punctuation marks for distinguishing sentences to clarify the meaning becomes not so vital in the web-chat English. The omission of punctuation can save a lot of time and make communication more convenient. In web-chat English, misspelling is common and can not only save a lot of time but also make the language humorous. The chatters are unwilling to correct them and gradually they are accepted by more and more people. Compared with the traditional English, web-chat English involves the creative spelling to make the language much easier and more compact. This way has two benefits: One is for rapid typing; the other is for humorous and interesting style. Here are examples (see Example 4):

Example (4) gr8t (great); 4wards (forwards); B4 (before); 4u (for you)

As we all know, the language appears just for the requirement of people's communication. Language makes people's life more wonderful. The foundation of its popularity lies in the social communication, while the direct reason is the improvement of the Internet. The Internet has brought many changes in our life. It breaks the limitation of distance in communication, which makes the world become much smaller than before. Due to the Internet, information is becoming the main power of social development. The rhythm of people's life and work becomes fast. 


\section{Features of Web-Chat English at Lexical Level}

Words are always the most active parts in language change. A large number of new words are unique to the Internet, which is one of the most outstanding features of web-chat English. Web-chat English, as a member of language family, is mostly characterized by its words. People who seldom surf the Internet may be confused by those s words. However, if you pay more attention to the new words, you will find some features as follows.

Abbreviation is also called clipping, which is always used when people chat online. Abbreviation can be divided into several types in web-chat English. First, front clipping which can be used as the deletion at the beginning of the word, like " U" (you), "Ur" (your), "M" (am), and "K" (ok). So "r u k?" in web-chat English stands for "Are you ok?". Second, middle clipping means the deletion at the middle of the word in web-chat English. Here are some examples, "b/c" (because), "wat" (what), and "Ppl" (people). Third, end clipping is the deletion at the end of the word, such as "b" (be), "o" (oh), "edu" (education), and "nick" (nickname). Fourth, middle and end clipping can be used as the deletion in the middle and end of the word. Examples are "pvt" (private), "b/t" (between), and "pls" (please).

Just as abbreviation, acronym is also frequently used in the chatting room. In a narrow sense, "acronym is made up from the first letters of the name of an organization" (HU, 2006), such as WB (World Bank) and CIA (Central Intelligence Agency). In a broad sense, it is formed by picking the initial letters of words, phrases, or even sentences. In fact, acronym is one of the most remarkable features in web-chat English, which can really save a great deal of time. It can be used not only in words, short phrases, but also in short sentences. Here is an example of acronym in the chatting room: "Asl" is short for "age, sex, and location". It is made up of the first letter of the three words. More examples are as follows (see Examples 5-6):

Example (5) BTW: by the way, TY: thank you, OMG: oh my God.

Example (6) ASAP: as soon as possible, VG: very good, CUL: see you later.

Compounding, a common way of creating news words, is the formation of new words by joining two or more bases. Words formed in this way are called as compounds. In chatting room, compounds are widely used and always divided into these three types.

(1) Noun compounds:

Noun + noun: key + board $\rightarrow$ keyboard, fire + wall $\rightarrow$ firewall, home + page $\rightarrow$ homepage

Adjective + noun: soft + ware $\rightarrow$ software, free + box $\rightarrow$ freebox, small + talk $\rightarrow$ smalltalk

Verb + noun: scroll + bar $\rightarrow$ scrollbar, chat + room $\rightarrow$ chatroom, play + boy $\rightarrow$ playboy

Noun + adverb: desk + top $\rightarrow$ desktop, lap + top $\rightarrow$ laptop

Adverb + noun: off + line $\rightarrow$ offline, on + line $\rightarrow$ online

(2) Verb compounds:

Verb + adverb: $\log +$ in $\rightarrow$ login, $\log +$ off $\rightarrow \operatorname{logoff}$, sign + up $\rightarrow$ signup

Adverb + verb: down + load $\rightarrow$ download, up + load $\rightarrow$ upload

Noun + verb: brain + wash $\rightarrow$ brainwash, lip + read $\rightarrow$ lipread

(3) Adjective compounds:

Noun + adjective: net + wise $\rightarrow$ netwise

Blending is a method of word-formation in which a new word is formed from two words, involving both parts of them. As a result, the new words combine the sounds and meanings of the original words. This method of 
word-formation is very popular in the chatting room and has such types below:

(1) head + word

emoticon $=$ emotion + icon; autoindustry $=$ automobile + industry

(2) head + tail

netizen $=$ network + citizen

infobahn $=$ information + autobahn

Chinglish $=$ Chinese + English

smog $=$ smoke + fog

(3) word + head

Webcam $=$ web + camera

Videophone $=$ video + telephone

Masscult $=$ mass + culture

(4) head + head

Telco $=$ telephone + company

gox $=$ gaseous + oxygen

(5) word + tail

Netcast $=$ net + broadcast

Radiogram $=$ radio + telegram

Emotion icons are widely used in the chatting room as a way to express one's feeling. Unlike the words, those special symbols look very vivid and are much easier to be accepted. Accompanied by the development of the Internet, the use of emotion icons is becoming popular in the chatting room.

Among them, the most commonly used emotions are as follows:

:-) - happiness, humor

:-O — shocked, amazed

:-( - sadness, displeasure

$\therefore($ - crying

:-) -winking

$:-]$ - sarcastic

:-) - smile

:-e — disappointed

$:-\{$ - angry

:-D - hearty laughing

\section{Features of Web-Chat English at Syntactic Level}

Syntax is about principles of forming and understanding correct English sentences. It studies the way words, phrases, and clauses are ordered and formally grouped, and the way these function in sequence. Syntactically, casual conversations in the chatting room tend to exhibit striking characteristics: the most important being simple and direct syntax. In English, length of sentences is diverse and varied, of which short sentence and long sentence are the two common ones. They have their own traits respectively. Short sentence is easy to be organized and understood. It is mostly used on spoken and informal occasions. Long sentence can express more complex concepts. Through the detailed description, long sentence could make the argument in depth and show the fact sufficiently, which is always seen on formal occasions. As a result, short and simple sentences are used frequently in our daily communication, which gives an impression of brevity to listeners. According to the data collected from the Internet, short and simple sentences account for $86.5 \%$ of the total number of sentences, 
occupying the dominant position in the chatting room. Long sentences are very rare. The reason is that online chatting is a kind of real-time conversation. It requires the interlocutors to make instant responses. Apart from the typing speed, the application of short sentences meets the requirement. Oppositely, the use of long sentence will lead to time delays that may be unacceptable for communication which attempts to work in a conversational mode (Werry, 1996). Therefore, it is not surprising that one- or two-word sentences appear so often in the chatting room.

The chatters on the Internet are always strangers to each other. To start and develop a conversation, they need to get more information about each other, like "a/s/ll" (age/sex/location). In such cases, a large amount of interrogative sentences are used in the chatting room. One person asks questions and the other gives answers. This mode of communication can not only offer you the basic information about the other and make the conversation going, but also give you the information you want.

To the exclamatory sentences in the chatting room, users aim at expressing their strong emotions on something. For the listeners, exclamatory sentences show your close attention to the speaker's information and your active participation in the conversation. The omission is a common phenomenon of web-chat English in which the omission of subject is a vivid characteristic (Farfeleder, 2000). The online chat usually involves two parties. In the face-to-face conversation, the subject pronouns are always "I" and "You". Unlike our traditional English, the web-chat English pays more attention to time-saving. Without the subject, the sentence meaning is still complete and can be understood by each other.

Here is a short conversation in the chatting room (see Example 7):

Example (7) A: a/s/l?

B: 25/male/New York. and $\mathrm{u}$ ?

A: 23/female/Washington. glad to see $u$.

B: me too, want to make friends with $u$.

A: Thank u. glad to be ur friend.

In this conversation, the subject pronoun "I" is omitted. However, the sentence meaning is still unchanged. It is obvious that on the premise of time-saving principle, every strategy can be adopted to reduce the complexity in the conversation if it is necessary. In addition, verbs and objects sometimes are also omitted in web-chat English.

\section{Impact of Web-Chat English}

Since the Internet becomes more and more popular, the number of people who have frequent access to the Internet and communicate through it explodes at an amazing rate, and web-chat English will be used more widely and frequently online. Among all users of web-chat English, young people stay the center position. They are so energetic and likely to be influenced by the Internet and the new language variety. Because of the chatting room, the way of making friends becomes much broader than before. Moreover, the chatting room gives the young people enough freedom that they need. That is the reason why online chat is so popular among young groups. Besides the young people, the middle-aged and the old groups are more or less influenced by web-chat language, because our life cannot be separated from the Internet and communication. That is why we say web-chat English is not a technical but a social production. If you take a notice of the English we use, it is easy to find the impacts of web-chat English on our daily communication. 
As a new variety of English, web-chat English not only brings pleasure and convenience to people's life, but also changes the language itself in many aspects like vocabulary. Many new words in web-chat English go into our daily communication. They can not only express our ideas vividly but also make expressions more interesting and humorous. The emergence of the new words leads to more topics in conversation. A good knowledge of these new words can also help us make full use of the resources on the Internet. Another impact is the sentence we use on the Internet. Web-chat English has changed the way we speak. More simple and short sentences are used in conversation, which are easy, brief, and concise to understand. Meanwhile, grammar seems to be not so important as before. The language is getting closer to life, and its application is becoming more flexible.

The rise of web-chat English is also a serious threat to the traditional English. The former is flexible and changeable, while the latter is comparatively regular. Compared with the traditional English, the web-chat English gets closer to life. In our daily communication, people, especially the young, pay more attention to the brevity and creativeness and ignore the language rules. The brevity and creativeness are also their excuses of making mistakes. The phenomenon probably leads to the indulgence of faults. What is worse, it may cause some old words disappear gradually. New words are of creativity, while old ones are meaningful. The simple and short sentences are easy and compact, while the long sentences are beautiful and charming. To English learners, the traditional English plays the important role in the study. Web-chat English is the supplement and modification of it.

In addition, for the people who are addicted to the Internet, they are used to making a conversation in the chatting room and are always active in it. However, they live in the virtual space and are not active in the daily communication. This situation is common in young groups which is harmful to their physical and mental development.

Through the above discussion, the readers may have a general idea of the influence of web-chat English. There are both advantages and disadvantages. The popularity of web-chat English enriches and perfects the English language. This new variety of language stands for the new power and brings energy to our life. Meanwhile, it is also a great challenge for English learners to have a comprehensive understanding of English.

\section{References}

CHEN, L. (2002). Network language: Hypothesized world message sign. Journal of Liaoning Normal University (Social Sciences Edition), 25(1), 82-85.

Cherny, L. (1999). Conversation and community: Chat in a virtual world. Standford, C.A.: CSLI Publications.

Cristal, D. (2001). Language and the internet (pp. 22-23). Cambridge. New York: Cambridge University Press.

Farfeleder, S. (2000). Analysis of chat communication. Journal of University of Salzburg, 3, 16.

HU, Z. L. (2006). An introduction to linguistics. Beijing: Beijing University Press.

Internet World Stats. (2011). World internet users and population stats [EB/OL]. Retrieved from http://www.Internetworldstats.com/stats. htm

Milroy, J. (1992). Linguistic variation and change (pp. 16-21). Oxford : Oxford Blackwell.

QI, Y. C. (1985). An introduction to linguistics. Shanghai: Shanghai Foreign Language Education Press.

Werry, C. C. (1996). Linguistic and interactional features of internet relay chat (pp. 47-50). Amsterdam: John Benjamins Publishing Company.

ZHANG, Y. H. (2010). Grammatical and pragmatic research on web language. Shanghai: Academia Press.

ZHU, Y. (1999). English and society. Hefei: Anhui Universtity Press. 